PROCEEDINGS OF THE

AMERICAN MATHEMATICAL SOCIETY

Volume 134, Number 10, October 2006, Pages 2951-2955

S 0002-9939(06)08323-7

Article electronically published on May 1, 2006

\title{
MAXIMALITY OF SUMS OF TWO MAXIMAL MONOTONE OPERATORS
}

\author{
JONATHAN M. BORWEIN
}

(Communicated by N. Tomczak-Jaegermann)

\begin{abstract}
We use methods from convex analysis, relying on an ingenious function of Simon Fitzpatrick, to prove maximality of the sum of two maximal monotone operators on reflexive Banach space under weak transversality conditions.
\end{abstract}

\section{INTRODUCTION AND PRELIMINARIES}

The central result of this paper, Theorem 5, marries recent work by Simons and Zalinescu [16] with additional convex analysis to provide an accessible short proof of the maximality of the sum of two maximal monotone operators.

Recall that the domain of an extended valued convex function, denoted dom $(f)$, is the set of points with value less than $+\infty$, and that a point $s$ is in the core of a set $S$ (denoted by $s \in$ core $S$ ) provided that $s$ lies in $S$ and $X=\bigcup_{\lambda>0} \lambda(S-s)$. For a concave function $g$, we use $\operatorname{dom} g=\operatorname{dom}(-g)$. Recall that $x^{*} \in X^{*}$ is a subgradient of $f: X \rightarrow(-\infty,+\infty]$ at $x \in \operatorname{dom} f$ provided that $f(y)-f(x) \geq\left\langle x^{*}, y-x\right\rangle$. The set of all subgradients of $f$ at $x$ is called the subderivative or subdifferential of $f$ at $x$ and is denoted $\partial f(x)$. We use the convention that $\partial f(x)=\emptyset$ for $x \notin \operatorname{dom} f$. We shall need the indicator function $\iota_{C}(x)$ which is zero for $x$ in $C$ and $+\infty$ otherwise, the Fenchel conjugate $f^{*}\left(x^{*}\right):=\sup _{x}\left\{\left\langle x, x^{*}\right\rangle-f(x)\right\}$ and the infimal convolution $f^{*} \odot \frac{1}{2}\|\cdot\|_{*}^{2}\left(x^{*}\right):=\inf \left\{f^{*}\left(y^{*}\right)+\frac{1}{2}\left\|z^{*}\right\|_{*}^{2}: x^{*}=y^{*}+z^{*}\right\}$. When $f$ is convex and closed and $x$ is in the domain of $f, x^{*} \in \partial f(x)$ exactly when $f(x)+f^{*}\left(x^{*}\right)=\left\langle x, x^{*}\right\rangle$.

We say a multifunction $F: X \mapsto 2^{X^{*}}$ is monotone provided that for any $x, y \in X$, $x^{*} \in F(x)$ and $y^{*} \in F(y)$,

$$
\left\langle y^{*}-x^{*}, y-x\right\rangle \geq 0
$$

and we say that $T$ is maximal monotone if its graph is not properly included in any other monotone graph. The subdifferential of a convex lower semicontinuous (lsc) function on a Banach space is a typical example of a maximal monotone multifunction (see [4, 6, 13] wherein other notation and usage may also be followed upon). Indeed we reserve the notation $J$ for the duality map

$$
J(x):=\frac{1}{2} \partial\|x\|^{2}=\left\{x^{*} \in X^{*}:\|x\|^{2}=\left\|x^{*}\right\|^{2}=\left\langle x, x^{*}\right\rangle\right\} .
$$

Received by the editors January 22, 2005 and, in revised form, April 27, 2005.

2000 Mathematics Subject Classification. Primary 47H05, 46N10, 46A22.

Key words and phrases. Monotone operators, convex analysis, Sandwich theorem, Fenchel duality, sum theorem.

This research was supported by NSERC and by the Canada Research Chair Program.

(C)2006 American Mathematical Society Reverts to public domain 28 years from publication 
Further applications and more extended discussion of the techniques in this note can be found in [1].

Proposition 1 (4, 6, 13]). For a closed convex function $F$, let $f_{J}:=f+\frac{1}{2}\|\cdot\|^{2}$. Then $f_{J}^{*}=\left(f+\frac{1}{2}\|\cdot\|^{2}\right)^{*}=f^{*} \odot \frac{1}{2}\|\cdot\|_{*}^{2}$ is everywhere continuous. Also,

$$
v^{*} \in \partial f(v)+J(v) \Leftrightarrow f_{J}^{*}\left(v^{*}\right)+f_{J}(v)-\left\langle v, v^{*}\right\rangle \leq 0 .
$$

For any monotone mapping $T$, we associate the Fitzpatrick function introduced by Simon Fitzpatrick in [8] but then neglected for many years until re-popularized in papers by Penot [10, Buracik-Svaiter [7, and others. Some more history may be found in [1]. Fitzpatrick's function is

$$
F_{T}\left(x, x^{*}\right):=\sup \left\{\left\langle x, y^{*}\right\rangle+\left\langle x^{*}, y\right\rangle-\left\langle y, y^{*}\right\rangle: y^{*} \in T(y), y \in \operatorname{dom} T\right\},
$$

which is clearly lower semicontinuous and convex as an affine supremum. Moreover,

Proposition 2 ([8, 6]). For a maximal monotone operator $T$

$$
F_{T}\left(x, x^{*}\right) \geq\left\langle x, x^{*}\right\rangle
$$

with equality if and only if $x^{*} \in T(x)$.

We recall the version of the Hahn-Banach theorem we need:

Theorem 3 (Hahn-Banach Sandwich, 4, 6, 13]). Suppose $f$ and $-g$ are proper extended real-valued lsc convex functions on a Banach space $X$ and that $f(x) \geq$ $g(x)$, for all $x$ in $X$. Assume that

$$
0 \in \text { core }(\operatorname{dom}(f)-\operatorname{dom}(g)) \text {. }
$$

Then there is a continuous linear function $\lambda$ such that

$$
f(x)-g(y) \geq\langle\lambda, x-y\rangle,
$$

for all $x \in \operatorname{dom} f, y \in \operatorname{dom}-g$ in $X$.

Proof. The value function $h(u):=\inf _{X} f(x)-g(x-u)$ is convex. It is continuous at 0 -indeed the constraint qualification and semi-continuity of the data force $h$ to be bounded above around zero-by a Baire category type argument [17, 14, 6]. Hence there is some $-\lambda \in \partial h(0)$. This provides the linear part of the asserted affine separator. Indeed, we have

$$
f(x)-g(u-x) \geq h(u)-h(0) \geq \lambda(u-0),
$$

as required.

The next result, implicit in the literature [14, avoids needing to renorm a reflexive space to have a single-valued duality map with a single-valued inverse.

Proposition 4 (14, 11, 12, 6]). A monotone multifunction $T$ is maximal if and only if the mapping $T(\cdot+w)+J$ is surjective for all $w$ in $X$. [When $J$ and $J^{-1}$ are both single valued, a monotone mapping $T$ is maximal if and only if $T+J$ is surjective.]

Proof. We prove the "if" part. The "only if" part is completed in Corollary 7 Assume $\left(w, w^{*}\right)$ is monotonically related to the graph of $T$. By hypothesis, we may solve $w^{*} \in T(x+w)+J(x)$. Thus $w^{*}=t^{*}+j^{*}$, where $t^{*} \in T(x+w), j^{*} \in J(x)$.

$$
0 \leq\left\langle w^{*}-t^{*}, w-(w+x)\right\rangle=-\left\langle w^{*}-t^{*}, x\right\rangle=-\left\langle j^{*}, x\right\rangle=-\|x\|^{2} \leq 0 .
$$


Hence $x=0, j^{*}=0$, and we are done.

The refined equivalence when $J, J^{-1}$ are single-valued may be found in [14, Thm. 10.6].

\section{THE MAIN RESULT}

We now prove our asserted result whose proof - originally very hard and due to Rockafellar [12] - has been revisited over many years culminating in [14, 7, 10, 16, 6], among others. The proof we give is perhaps the first to avoid using either renorming [12] or some preliminary minimax arguments [14]:

Theorem 5. Let $X$ be any reflexive space with given norm. Let $T$ be maximal monotone and $f$ closed and convex. Suppose that

$$
0 \in \operatorname{core}\{\operatorname{conv} \operatorname{dom}(T)-\operatorname{conv} \operatorname{dom}(\partial f)\} .
$$

Then

(a) $\partial f+T+J$ is surjective;

(b) $\partial f+T$ is maximal monotone;

(c) $\partial f$ is maximal monotone.

Proof. (a) As in [16], we consider the Fitzpatrick function $F_{T}\left(x, x^{*}\right)$ and further introduce $f_{J}(x):=f(x)+1 / 2\|x\|^{2}$. Let $G\left(x, x^{*}\right):=-f_{J}(x)-f_{J}^{*}\left(-x^{*}\right)$. Observe that

$$
F_{T}\left(x, x^{*}\right) \geq\left\langle x, x^{*}\right\rangle \geq G\left(x, x^{*}\right)
$$

pointwise thanks to Proposition 2, and the Fenchel-Young inequality: for any function $f(x)+f^{*}\left(x^{*}\right) \geq\left\langle x, x^{*}\right\rangle, \forall x, x^{*}$. Now, the (CQ)

$$
0 \in \operatorname{core}\{\operatorname{conv} \operatorname{dom}(T)-\operatorname{conv} \operatorname{dom}(\partial f)\}
$$

assures that the Sandwich theorem applies. Indeed, by Proposition $1, f_{J}^{*}$ is everywhere finite and in consequence zero is in the core of $\operatorname{dom} F_{T}-\operatorname{dom} G$.

Then there are $w \in X$ and $w^{*} \in X^{*}$ such that

$$
F_{T}\left(x, x^{*}\right)-G\left(z, z^{*}\right) \geq w\left(x^{*}-z^{*}\right)+w^{*}(x-z)
$$

so that for all $x^{*} \in T(x), x \in \operatorname{dom}(T)$ and for all $z^{*}, z$ we have

$$
\left\langle x^{*}-w^{*}, x-w\right\rangle+\left[f_{J}(z)+f_{J}^{*}\left(-z^{*}\right)+\left\langle z, z^{*}\right\rangle\right] \geq\left\langle w^{*}-z^{*}, w-z\right\rangle .
$$

Now use the fact that $-w^{*} \in \operatorname{dom}\left(\partial f_{J}^{*}\right)$, by Proposition 1, to deduce that for some $v,-w^{*} \in \partial f_{J}(v)$ and so

$$
\left\langle x^{*}-w^{*}, x-w\right\rangle+\left[f_{J}(v)+f_{J}^{*}\left(-w^{*}\right)+\left\langle v, w^{*}\right\rangle\right] \geq\left\langle w^{*}-w^{*}, w-v\right\rangle=0 .
$$

The second term on the left is zero, and so $w^{*} \in T(w)$ by maximality. Substitution of $x=w$ and $x^{*}=w^{*}$ in (11), and rearranging yields

$$
\left\langle w^{*}, w\right\rangle+\left\{\left\langle-z^{*}, w\right\rangle-f_{J}^{*}\left(-z^{*}\right)\right\}+\left\{\left\langle z,-w^{*}\right\rangle-f_{J}(z)\right\} \leq 0,
$$

for all $z, z^{*}$. Taking the supremum over $z$ and $z^{*}$ produces $\left\langle w^{*}, w\right\rangle+f_{J}(w)+$ $f_{J}^{*}\left(-w^{*}\right) \leq 0$. This shows $-w^{*} \in \partial f_{J}(w)=\partial f(w)+J(w)$ on using the sum formula for subgradients, implicit in Proposition 1 .

Thus, $0 \in\left(T+\partial f_{J}\right)(w)$ and, since all range translations of $T+\partial f$ may be used, $\partial f+T+J$ is surjective, which completes (a). Additionally, since all domain translations may be used, $\partial f+T$ is maximal by the easy part of Proposition 4 , which yields (b). 
Finally, setting $T \equiv 0$ we recover the reflexive case of the maximality for a lsc convex function $\partial f$ which is (c).

Note that we have exploited the beautiful inequality

$$
F_{T}\left(x, x^{*}\right)+f(x)+f^{*}\left(-x^{*}\right) \geq 0 \quad \forall x \in X, x^{*} \in X^{*},
$$

valid for any maximal monotone $T$ and any convex function $f$.

\section{SOME COROLlaries}

We first recover the so-called Brezis-Attouche theorem:

Corollary 6 ([14]). The sum of two maximal monotone operators $T_{1}$ and $T_{2}$ is maximal monotone if $0 \in \operatorname{core}\left[\operatorname{conv} \operatorname{dom}\left(T_{1}\right)-\operatorname{conv} \operatorname{dom}\left(T_{2}\right)\right]$.

Proof. Theorem 5 applies to the maximal monotone mapping $T(z):=\left(T_{1}(x), T_{2}(y)\right)$ and the indicator function $f(x, y)=\iota_{\{x=y\}}$. Finally, check that the given transversality condition implies the needed (CQ). We obtain that $T+J_{X \otimes X}+\partial \iota_{\{x=y\}}$ is surjective. Thus, so is $T_{1}+T_{2}+2 J$, and we are done.

We next recover the Rockafellar-Minty surjectivity theorem:

Corollary 7. A maximal monotone on a reflexive space has range $(T+J)=X^{*}$.

Proof. Let $f \equiv 0$ in Theorem 5

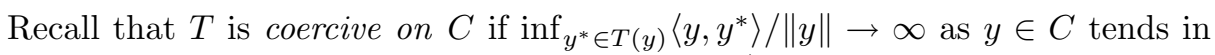
norm to infinity, and using the convention that $\inf \emptyset=+\infty$.

A variational inequality requests a solution $y \in C$ and $y^{*} \in T(y)$ to

$$
\left\langle y^{*}, x-y\right\rangle \geq 0 \quad \forall x \in C .
$$

We denote the variational inequality by $V(T ; C)$.

Corollary 8. Suppose $T$ is maximal monotone on a reflexive Banach space and is coercive on the closed convex set $C$. Suppose also that $0 \in \operatorname{core}(C-\operatorname{conv} \operatorname{dom}(T))$. Then $V(T, C)$ has a solution.

Proof. Without loss, we assume 0 lies in $C$. Let $f:=\iota_{C}$, the indicator function. For $n=1,2,3 \cdots$, let $T_{n}:=T+J / n$. We solve

$$
0 \in\left(T_{n}+\partial \iota_{C}\right)\left(y_{n}\right)=\left(\left(T+\partial \iota_{C}\right)+\frac{1}{n} J\right)\left(y_{n}\right)
$$

and take limits as $n$ goes to infinity. More precisely, we observe that using Theorem 5] we find $y_{n}$ in $C$, and $y_{n}^{*} \in T\left(y_{n}\right), j_{n}^{*} \in J\left(y_{n}\right) / n$ with

$$
\left\langle y_{n}^{*}, x-y_{n}\right\rangle \geq-\left\langle j_{n}^{*}, x-y_{n}\right\rangle \quad \forall x \in C .
$$

Then coercivity (of $T+\partial \iota_{C}$ ) implies that $\left\|y_{n}\right\|$ remains bounded and so $j_{n}^{*} \rightarrow 0$. On taking a subsequence we may assume $y_{n} \neg y, y_{n}^{*} \rightarrow y^{*}$. Since $T$ is demi-closed [6], it follows that $y^{*} \in T(y)$ and that

$$
\left\langle y^{*}, x-y\right\rangle \geq 0 \quad \forall x \in C .
$$

Letting $C=X$ we deduce:

Corollary 9. Every coercive maximal monotone multifunction on a Banach space is surjective if (and only if) the space is reflexive. 
Proof. To complete the proof we recall that, by James' theorem, surjectivity of $J$ is equivalent to reflexivity of the corresponding space.

Details of these and other corollaries are to be found in [1].

Finally, warm thanks are due to Jean-Paul Penot and to Jim Zhu for their very helpful comments about this note.

\section{REFERENCES}

[1] Borwein J. M., "Maximal montonicity via convex analysis," to appear in the Fitzpatrick memorial issue of J. Convex Analysis, 2006.

[2] Borwein J. M. ANd FitzPatrick, S., "Local boundedness of monotone operators under minimal hypotheses," Bull. Australian Math. Soc., 39 (1988), 439-441. MR0995141 (90c:47093)

[3] J.M. Borwein, S. Fitzpatrick and J. VanderwerfF, "Examples of convex functions and classifications of normed spaces," Journal of Convex Analysis, 1 (1994), 61-73. MR 1326942 (96e:46012)

[4] Bonwein J. M. \& Lewis A. S., Convex Analysis and Nonlinear Optimization: Theory and Examples, Springer-Verlag, New York, 2000. MR.1757448 (2001h:49001)

[5] Borwein J. M. \& ZHU Q. J., "A survey of subdifferential calculus with applications," Nonlinear Analysis, TMA, 38 (1999), 687-773. MR.1710152 (2000j:49024)

[6] Borwein J. M. \& Zhu Q. J., Techniques of Variational Analysis: an Introduction, CMS Books, Springer-Verlag, 2005. MR2144010

[7] Burachik, R.S., \& Svaiter, B.F., Maximal monotonicity, conjugation and the duality product," Proc AMS, 131 (2003), 2379-2383. MR1974634 (2004a:49037)

[8] Fitzpatrick, S. "Representing monotone operators by convex functions," Workshop/Miniconference on Functional Analysis and Optimization (Canberra, 1988), 59-65, Proc. Centre Math. Anal. Austral. Nat. Univ., 20, Austral. Nat. Univ., Canberra, 1988. MR1009594 (90i:47054)

[9] Borwein J. M. \& ZhU Q. J., A survey of subdifferential calculus with applications, Nonlinear Analysis, TMA, 38 (1999), 687-773. MR.1710152 (2000j:49024)

[10] Penot J.P., "The relevance of convex analysis for the study of montonicity," Nonlinear Anal, Theory, Methods, Appl., 58 (2004), 855-871. MR2086060 (2005g:49026)

[11] Rockafellar R. T., "Local boundedness of nonlinear monotone operators," Mich. Math. J., 16 (1969), 397-407. MR0253014(40:6229)

[12] Rockafellar R. T., "On the maximality of sums of nonlinear monotone operators," Trans. Amer. Math. Soc., 149 (1970), 75-88. MR0282272 (43:7984)

[13] Rockafellar R. T., Convex Analysis, Princeton University Press, Princeton, N.J., 1970. MR0274683(43:445)

[14] Simons, S., Minimax and Monotonicity, Lecture Notes in Mathematics, 1693, SpringerVerlag, Berlin, 1998. MR 1723737 (2001h:49002)

[15] Simons, S., "A new version of the Hahn-Banach theorem," Arch. Math. (Basel) 80 (2003), 630-646. MR1997529(2004j:46007)

[16] Simons, S. AND C. Zalinescu, "A new proof for Rockafellar's characterization of maximal monotone operators," Proc. Amer. Math. Soc., 132 (2004), 2969-2972. MR2063117 (2005f:47121)

[17] Verona, A. And Verona, M. E., "Regular maximal monotone operators and the sum rule," J. Convex Analysis, 7 (2000), 115-128. MR1773179 (2001h:47087)

Faculty of Computer Science, Dalhousie University, Halifax, Nova Scotia, Canada

E-mail address: jborwein@cs.dal.ca 